\title{
Evolutionary Analysis and Expression of Teleost Thy-1
}

\author{
ALEXANDER REUTER, ${ }^{* *}$ EDWARD MÁLAGA-TRILLO, ${ }^{1 *}$ ULRIKE BINKLE, ${ }^{1}$ \\ ERIC RIVERA-MILLA, ${ }^{1}$ ROSANNA BELTRE, ${ }^{2}$ YI ZHOU, ${ }^{2}$ MARTIN BASTMEYER, ${ }^{1}$ \\ and CLAUDIA A.O. STUERMER ${ }^{1}$
}

\begin{abstract}
Thy-1 is a developmentally regulated, immunoglobulin superfamily member (IgSF), glycosylphosphatidylinositol (GPI)-anchored cell surface glycoprotein expressed most strongly in neurons and lymphocytes. Thy-1 is expressed in all vertebrates and has been implicated in a variety of processes, including axon regeneration and transmembrane signaling, but its specific function remains elusive. A Thy-1-like molecule in teleost fish was recently identified, with evidence for its role in lipid-raft based signal transduction linked to optic nerve regeneration. For a better characterization of Thy-1, the evolutionary relationships between novel fish homologues and other vertebrate Thy-1s were analyzed. Although the sequence similarity between fish and mammals is very low, there appeared conservation of gene structure and disrupted but recognizable synteny. In addition, the detailed expression analysis of teleost Thy-1 showed nervous system Thy-1 mainly in sensory systems. Strong Thy-1 expression was detected in the youngest retinal ganglion cells and in some neurons in deeper retinal layers, probably amacrine cells. From the olfactory bulbs, Thy-1-positive cells extended axons into the telencephalon. The vagal lobe stained intensively as well as facial and glossopharyngeal lobes and nerves. Outside the CNS, skin cells, blood vessels, kidney macrophages, swim bladder, spleen, gut-associated nerve fibers and the palatal organ were labeled.
\end{abstract}

\section{INTRODUCTION}

$\mathbf{T}$ hy- 1 is a member of the immunoglobulin superfamily ${ }^{1,2}$ with a molecular weight of $18 \mathrm{kD}$. It is comprised of 110 amino acids and is localized to the surface of T lymphocytes and neurons by a GPI anchorage. Until now, homologous Thy- 1 gene sequences have been reported in mammals, ${ }^{3}$ birds, ${ }^{4}$ and more recently in teleosts, ${ }^{5}$ implying an ancient origin for this molecule that dates back to the early base of the vertebrate lineage. So far, no homologues have been identified in invertebrates, except early erroneous claims based on immunological evidence. ${ }^{6}$

The function of Thy- 1 remains elusive, despite being one of the most abundant glyco- proteins in mammalian neurons. Thy-1 has been implicated in $\mathrm{T}$ cell receptor-mediated and lipid raft based signal transduction.,8 Thy-1 was also suggested to play a role in cell adhesion $^{9,10}$ and inhibition of neurite outgrowth, ${ }^{11}$ but Thy-1 knockout mice did not show an increased capacity of regeneration ${ }^{12}$ or other apparent defects. The expression of Thy- 1 is developmentally regulated. ${ }^{13,14} \mathrm{Neu}^{-}$ rons express Thy-1 after they have migrated to their final position and begin to grow dendrites. ${ }^{15,16}$

The expression pattern of Thy- 1 in mammals varies among species. Although it is invariably expressed in brain, only rodents express it in lymphoid tissues. ${ }^{17-20}$ Thy-1 expression has been detected in kidney urothelium and visceral

\footnotetext{
${ }^{1}$ Department of Biology, Neurobiology, University of Konstanz, Konstanz, Germany.

${ }^{2}$ Division of Hematology/Oncology, Children's Hospital Boston and Dana-Farber Cancer Institute, Boston, Massachusetts.

*These authors contributed equally to this publication.

Supported by Deutsche Forschungsgemeinschaft (DFG) and Fonds der Chemischen Industrie (FCI). E.R-M. is a Deutscher Akademischer Austauschdienst (DAAD) fellow.
} 
smooth muscle, as well as in human but not in murine kidney tubules and blood vessels. In both species, expression can be detected in skin fibroblasts and connective tissues, but the function of Thy- 1 in these tissues remains unclear.

Recently, homologues of Thy-1 in goldfish and zebrafish were identified and its upregulation shown in regenerating goldfish optic nerve. ${ }^{5}$ Moreover, Thy- 1 is enriched on the regenerating axons throughout their path into the optic tectum ${ }^{21}$ and is associated with Reggie/ Flotillin proteins in lipid raft microdomains. ${ }^{22}$ In fact, Reggies were identified through coimmunoprecipitation with mAB M802 against fish Thy-1.5,21 This association with Reggierafts also occurs in Jurkat T cells and PC12 cells, where coclusters of Thy-1 and Reggies are distributed in a punctate pattern along the plasma membrane and into the PC12 cell neurites. The coclusters also are spatially condensed at cell contact sites and Jurkat $\mathrm{T}$ cell caps, ${ }^{7,22}$ suggesting that Thy-1 participates in the communication between cells.

Prerequisite for protein functional analyses in vivo, for instance via morpholino (antisense RNA) knockdown, is the accurate knowledge of the Thy- 1 gene and protein expression pattern in fish. Here, the evolutionary relationships between fish and mammalian homologues were analyzed, as well as its expression in adult and juvenile fish.

\section{MATERIALS AND METHODS}

\section{Immunohistochemistry}

MAB M802 against goldfish Thy-1 was applied to methanol-fixed goldfish cryosections at room temperature (RT) for 1 hour. The cells were washed and incubated with an Alexa 488coupled donkey anti-mouse secondary antibody and with 4',6-diamidino-2-phenylindole (DAPI) for nuclei staining for 1 hour at RT. The sections were mounted with Mowiol containing n-propylgallate as an antifading agent. The sections were analysed using a Zeiss Axioplan 2 fluorescence microscope.

\section{Retina and Tissue Preparation}

Goldfish were maintained in the central animal facilities (Tierforschungsanlage [TFA],
University of Konstanz). The optic nerves of adult goldfish (5-8 cm long) were transected under MS 222 anesthesia in compliance with animal welfare legislation. Retinae were isolated and attached to nylon membranes, placed on polylysine/laminin-coated coverslips, and incubated in F12 medium (with 12\% fetal calf serum) in a humidified chamber at $28^{\circ} \mathrm{C}$.

\section{Molecular Evolutionary and Genomic Analysis}

Previously obtained Thy-1 DNA and amino acid sequences were used to search public genome and EST databases for novel homologous genes in fish. Best matches were analyzed for consistent open reading frames (ORF). Multiple sequence alignments were initially performed with CLUSTALW (PAM-250 scoring matrix) at http://www.ebi.ac.uk/clustalw/ index.html, and further optimized manually based on previously reported Thy-structural information, ${ }^{2}$ as well as on secondary structure predictions at http://www.aber.ac.uk/ phiwww/prof/ by the Aberystwyth Computational Biology Group (University of Wales). Neighbor Joining phylogenetic trees were reconstructed with distance methods in Phylogenetic Analysis Using Parsimony (PAUP) v4.0d64 (Sinauer Associates, Massachusetts). Reliability of tree topologies was estimated by 1000 bootstrap replications. To determine synteny, the fish and mammalian genomic regions around Thy-1 genes were analyzed for gene content and order using GENSCAN at http:// genes.mit.edu/GENSCAN.html, and subsequent reciprocal BLAST searches at http:// www.ncbi.nlm.nih.gov/BLAST/, http://www. ensembl.org, and http://www.jgi.doe.gov/ genomes/index.html). Human, mouse, Takifugu, and zebrafish (assembly version 4, Zv4) genomic data were obtained from the corresponding databases at http://www.ensembl. org/. Zebrafish Thy-1 was mapped using a radiation hybrid panel as previously described. ${ }^{3}$

\section{RESULTS}

Evolutionary Relationships Among Vertebrate Thy-1 Genes

To establish whether functional homology between distantly related Thy-1 proteins could 


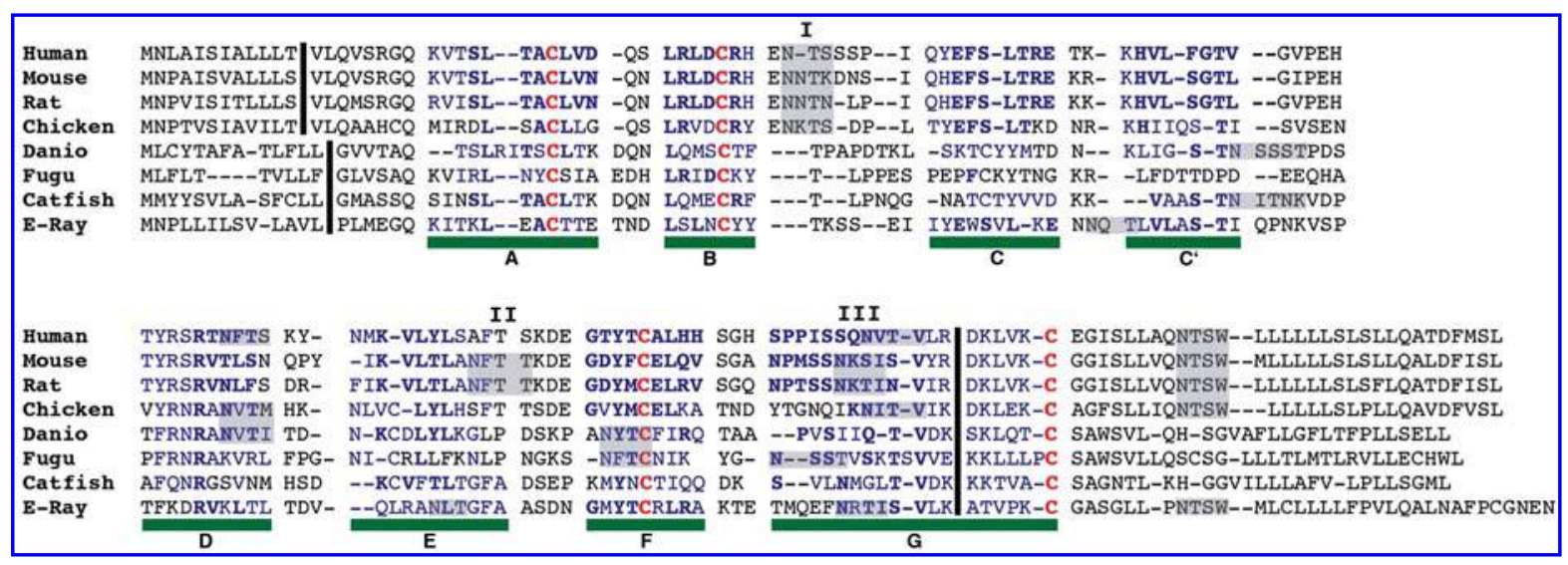

FIG. 1. Amino acid alignment of vertebrate Thy-1 molecules. Residues predicted to form $\alpha$-helical stretches are shown in blue (and bold when conserved), underscored by green horizontal bars, and named according to Williams and Gagnon"; predicted N-glycosylation sites are framed in grey boxes and mouse functional ones indicated with roman numerals; cysteines involved in disulfide bridge formation are shown in red; black vertical bars indicate the position of the exon boundaries in the gene.

be inferred from molecular conservation, we examined the degree of evolutionary variation between the amino acid sequences of fish Thy- 1 and those of mammals and birds.

Avian and mammalian Thy-1 molecules are highly conserved in sequence (about 65\% similarity, compared to the $>75 \%$ observed among mammalian sequences); importantly, distinctive structural/functional features are conserved between the Thy- 1 of birds and mammals, such as protein size, the location of a GPI-anchor, cysteine residues and $\mathrm{N}$-glycosylation sites (Fig. 1), suggesting a highly conserved function between the two classes of land vertebrates.

The situation in fish is highly contrasting. We extended our analysis by including the Thy- 1 amino acid sequence of zebrafish, ${ }^{5}$ as well as newly obtained sequences from Takifugu, catfish, and the cartilaginous electric ray. The sequences of electric ray (Acc. no. AJ276366) and catfish (Acc. no. BM027934) were obtained from ESTs by Genbank database searches, whereas the Takifugu sequence (Acc. No. BK005221) was assembled from the Takifugu genomic sequence (Acc. No.CAAB01000000). Since genomic data is publicly available for zebrafish and Takifugu (see Materials and Methods), we analyzed their gene structure, and found it to be the same as that of tetrapod Thy-1s, with the open reading frame (ORF) encoded in three exons. Using bioinformatic tools, we also could predict a consistent Ig folding for all fish Thy-1 homologues. Despite these general similarities, large differences between fish and mammalian molecules were also evident, such as a dramatic reduction in sequence similarity to an average of $20 \%$ and a slight variation in protein size, indicating rapid rates of molecular substitution and the occurrence of insertion/deletion events, respectively.

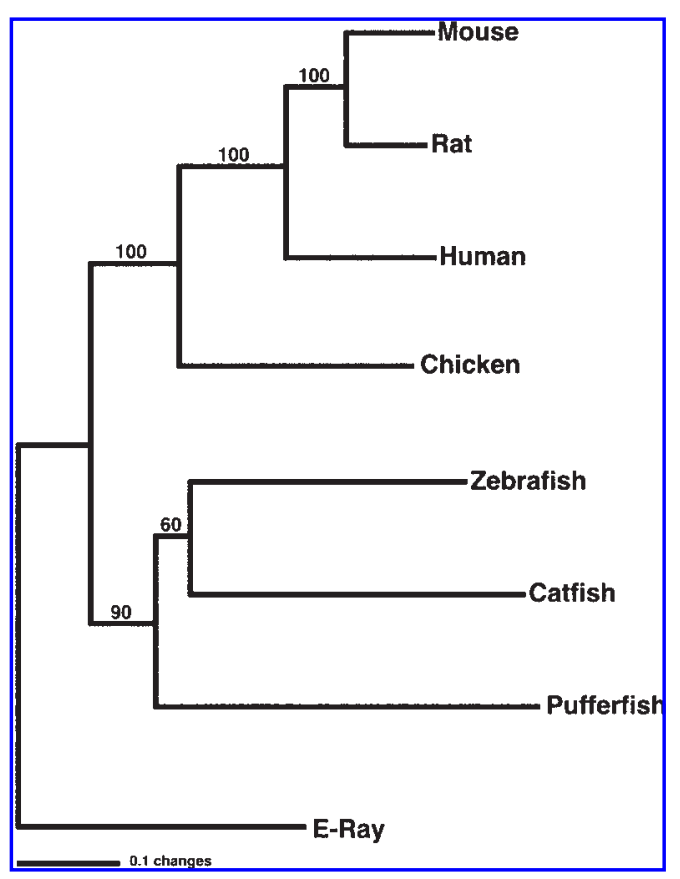

FIG. 2. Evolutionary relationships among vertebrate Thy-1 sequences, based on an optimized amino acid alignment. Numbers at nodes indicate bootstrap confidence values. 


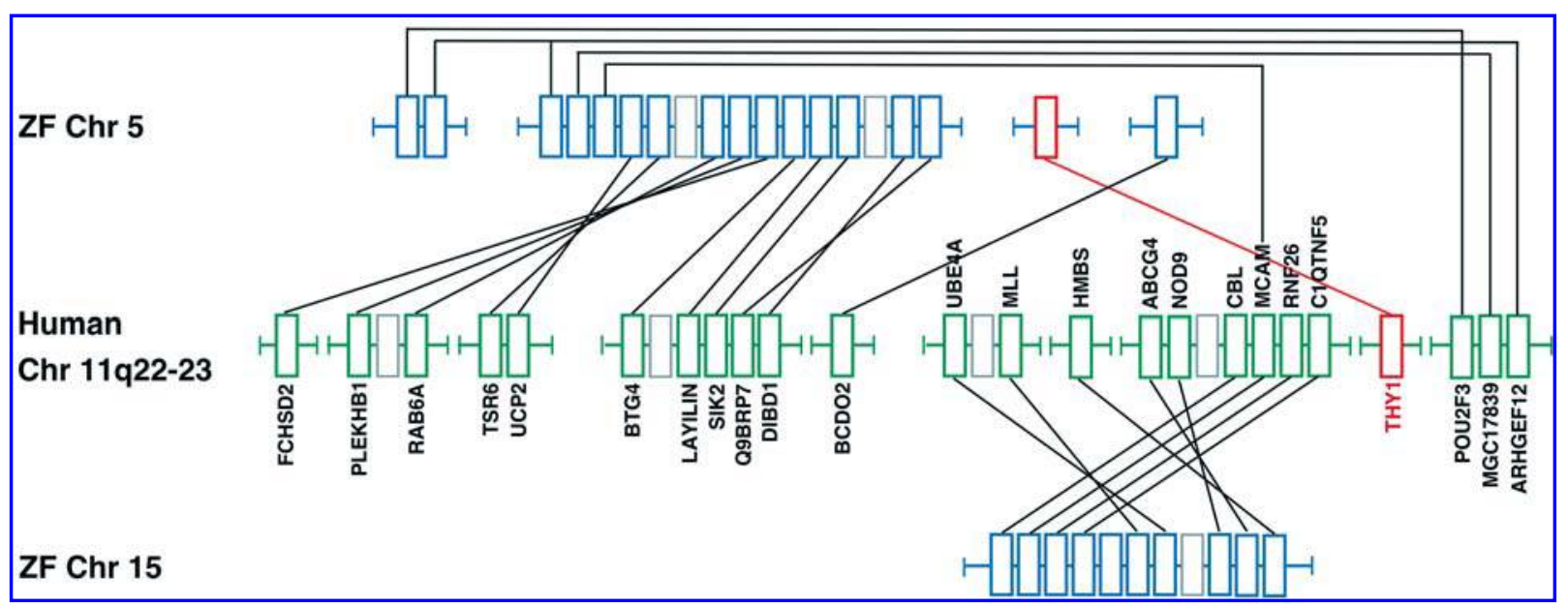

FIG. 3. Syntenic relationships between the human (green) and zebrafish (blue) genomic regions containing Thy1 genes (red). Colored and grey boxes represent relevant and unrelated genes, respectively; discontinuous lines indicate longer intervals with unrelated genes (mosaicism).

Closer inspection of sequences in an optimized alignment showed that the number and exact location of putative N-glycosylation sites varied markedly in all fish species considered, and was different from the invariant pattern of three sites found in mammalian and chick Thy-1 molecules (Fig. 1), indicating that fish produce somewhat differently modified Thy-1 glycoproteins than land vertebrates. However, the partial deglycosylation data of Deininger et al., ${ }^{5}$ which show three partially deglycosylated bands in addition to the fully deglycosylated form, suggest that although more putative glycosylation sites of variable location may be available in teleost sequences, the same number of functional sites is conserved between fish and mammals.

The phylogenetic tree in Figure 2 summarizes the evolutionary relationships between verte- brate Thy-1 proteins. With the electric ray at the root of the phylogram, two major clusters (bony fish and land vertebrates) are recovered, both of them internally structured according to the accepted taxonomic groupings. When comparing the branch lengths (genetic distances) in the tree, a remarkable difference emerges between fish and tetrapod Thy-1 molecules, namely the large genetic divergence between teleost sequences, indicating that bony fish homologues have accumulated amino acid substitutions at a higher rate than their tetrapod counterparts. We also assessed the differences between the fish and mammalian Thy-1 genomic regions, by analyzing the corresponding chromosomal segments in human chromosome 11q23, mouse chromosome 9B, zebrafish, and Takifugu, and by taking advantage of the publicly available

FIG. 4. Immunohistochemical localization of Thy-1 in the adult goldfish brain. M802 was detected by a Alexa488-coupled secondary AB (green), whereas the nuclei of the cells were stained by DAPI (blue). (A) Overview of a sagittal goldfish brain section. The boxes delineate the approximate position of photomicrographs $\mathbf{B}$ and $\mathbf{C}$. (B) Sagittal section of the bulbus olfactorius (BO) where groups of cells are labeled (arrows). (C) Sagittal section of the vagal lobe. The main sensory root (arrow) and the capsular sensory root (arrowhead) are labeled intensely. (D) Overview of a caudal goldfish vagal lobe cross section. The rectangle demarcates the approximate position of photomicrograph E. The caudal sensory root (large arrow) and the capsular sensory root (small arrows) are marked. (E) Caudal goldfish vagal lobe cross section. Label is concentrated at the caudal sensory root (arrow), the ventral motoneuron group of the vagus nerve (nXM, arrowhead) and the capsular sensory root (small arrows). (F) Overview of a central goldfish vagal lobe cross section. The rectangle demarcates the approximate position of photomicrograph G. Main sensory root (MSR), cutaneous sensory root (CSR), vagal lobe (LX), facial lobe (LVII), and glossopharyngeal lobe (LIX) are marked. (G) Central goldfish vagal lobe cross section. Positive immunoreactivity for Thy-1 can be detected in the MSR (arrowhead), CSR (arrow), and weaker in the facial and glossopharyngeal lobes. (H) Section of a goldfish vagal lobe labeled for Thy-1. Label is concentrated in layers II (capsular sensory root) and XII (MSR). (I) Cross section of a goldfish brain in the vagal lobe region. In addition to the staining in the vagal lobe (arrows), weaker label can be detected in facial (LVII) and glossopharyngeal lobe (LIX). Nuclei were counterstained with DAPI. Scale bars: $50 \mu \mathrm{m}$ for $\mathbf{H}, 300$ $\mu \mathrm{m}$ for $\mathbf{B}$, and $500 \mu \mathrm{m}$ for $\mathbf{C}, \mathbf{E}, \mathbf{G}$, and I . 


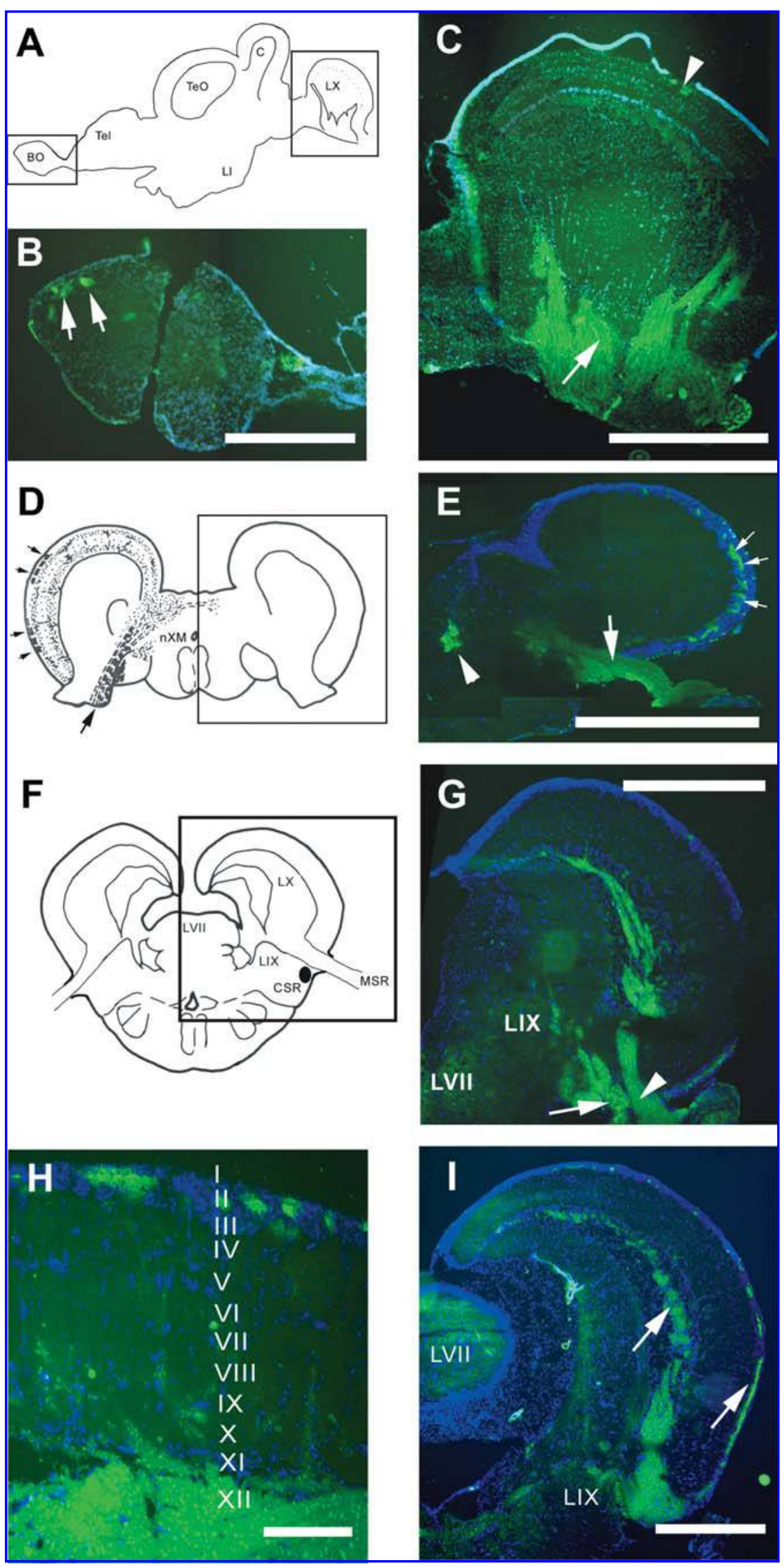

FIG. 4. 
bioinformatic databases (see Materials and Methods). In both teleost fish, we identified two main genomic regions containing relevant homologues of mammalian Thy- 1 gene neighbors. Figure 3 summarizes the zebrafish data; two chromosomes, 5 and 15, display overlapping segment homologies to the mammalian genomic region containing Thy- 1 and are likely to represent duplicated chromosomal blocks. Because their gene contents are complementary, they are likely to have undergone differential gene loss: only the block in chromosome 5 retains a Thy- 1 homologue, and its genomic localization was established by radiation hybrid mapping. Interestingly, two large blocks of genes known to be linked upstream of Thy-1 in humans were also found tightly linked but separated on zebrafish chromosomes 5 and 15 (Fig. 3 ), suggesting that the duplicated Thy-1 regions of fish underwent independent and extensive changes that disrupted the conserved syntenic relationships seen in mammals, possibly driving rapid molecular divergence.

\section{Thy-1 Expression in the Nervous System}

In agreement with the tissue distribution of Thy- 1 in most mammals and chicken, ${ }^{23}$ Thy- 1 is expressed most strongly in specific regions of the brain, especially sensory systems: neurons of the olfactory bulb (Fig. 4B), the VII, IX, and $X$ nerve and the vagal (Fig. $4 C$ ), as well as facial and glossopharyngeal lobes.

The teleost vagal lobe is a highly laminated structure, consisting of 16 histologically distinguishable layers. ${ }^{24-26}$ The 11 most external layers make up the sensory zone. Odd numbered layers contain neurons and even numbered layers axons. The sensory and the deeper motor zone are separated by two fiber layers. The two innermost motor zone layers are localized above the ependymal layer which faces the fourth ventricle. Besides a weak general staining, the anti-Thy-1 AB labeled intensely the main sensory root (MSR) of the vagus nerve that enters the vagal lobe ventrally (Figs. 4B, 4E, 4G). Axons in this root ascend dorsally, form the fibrous twelfth layer of the vagal lobe which, therefore, stains intensely (Figs. 4C, 4E, 4H, 4I). The capsular sensory root which courses tangentially along the superficial part of the vagal lobe and forms the second layer (Figs. 4E, 4G,
$4 \mathrm{H})$ is also strongly stained, and so are the caudal sensory root (Fig. 4E), the cutaneous sensory root (CSR, Fig. 4G), and the ventral motoneuron group of the vagus nerve (nXM, Figs. $4 \mathrm{D}, 4 \mathrm{E})$. The facial (LVII) and glossopharyngeal lobes (LIX), as well as the facial nerve are less intensely labeled than the vagal lobe (Fig. 4I). Although there was some staining in the brainstem, most likely the cutaneous sensory root of the vagus nerve, no Thy- 1 immunoreactivity was detected in the spinal cord. In more anterior regions of the brain, the olfactory bulbs contain Thy-1 positive cells which extend axons into the telencephalon (Figs. 4B, 5A). A typical feature of Thy- 1 in fish is its expression in endothelial cells of blood vessels (Fig. 5B) and capillaries in the brain, optic nerve, and retina (Fig. 5C). Here Thy-1 expression is particularly high at contact sites (marked by arrows).

In the retina of adult goldfish, Thy-1 expression is restricted to the youngest RGCs at the retinal margin and to new growing axons (Fig. 5D). Interestingly, RGCs at the retinal margin are only labeled in fish $>12 \mathrm{~mm}$ length whereas RGCs of fish smaller than that and larvae or embryos are not labeled. Yet, when the optic nerve was lesioned and RGC axons regenerate, all RGCs including the most central ones express Thy- 1 which failed to do so in the small fish (Fig. 5E). In addition, some neurons in deeper retinal layers, are labeled (Fig. 5F). Whole mounted embryos exhibited no Thy-1 immunoreactivity above background before a stage corresponding to the $48 \mathrm{~h}$ zebrafish larva, when staining of the skin becomes evident.

\section{Expression Pattern in Nonneuronal Tissues}

Outside the CNS, labeling is concentrated in epithelia such as the skin (data not shown), in the blood vessels-especially adjacent to the heart-and in the swim bladder (Fig. 6A). The intestine (Fig. 6B) shows strong staining in the smooth muscle cell layer. Strongly stained cells are detected in the intestine (Fig. 6B), the kidney tubules (Fig. 6C), the gill arches (Fig. $6 \mathrm{D})$, the oral cavity and the palatal organ (Fig. $6 \mathrm{E})$ which, due to the irregularity of their distribution and occurrence, most likely represent macrophages and, occasionally, sensory neurons of the gustatory system. Also labeled are the cartilageous structures of the palatal plate 


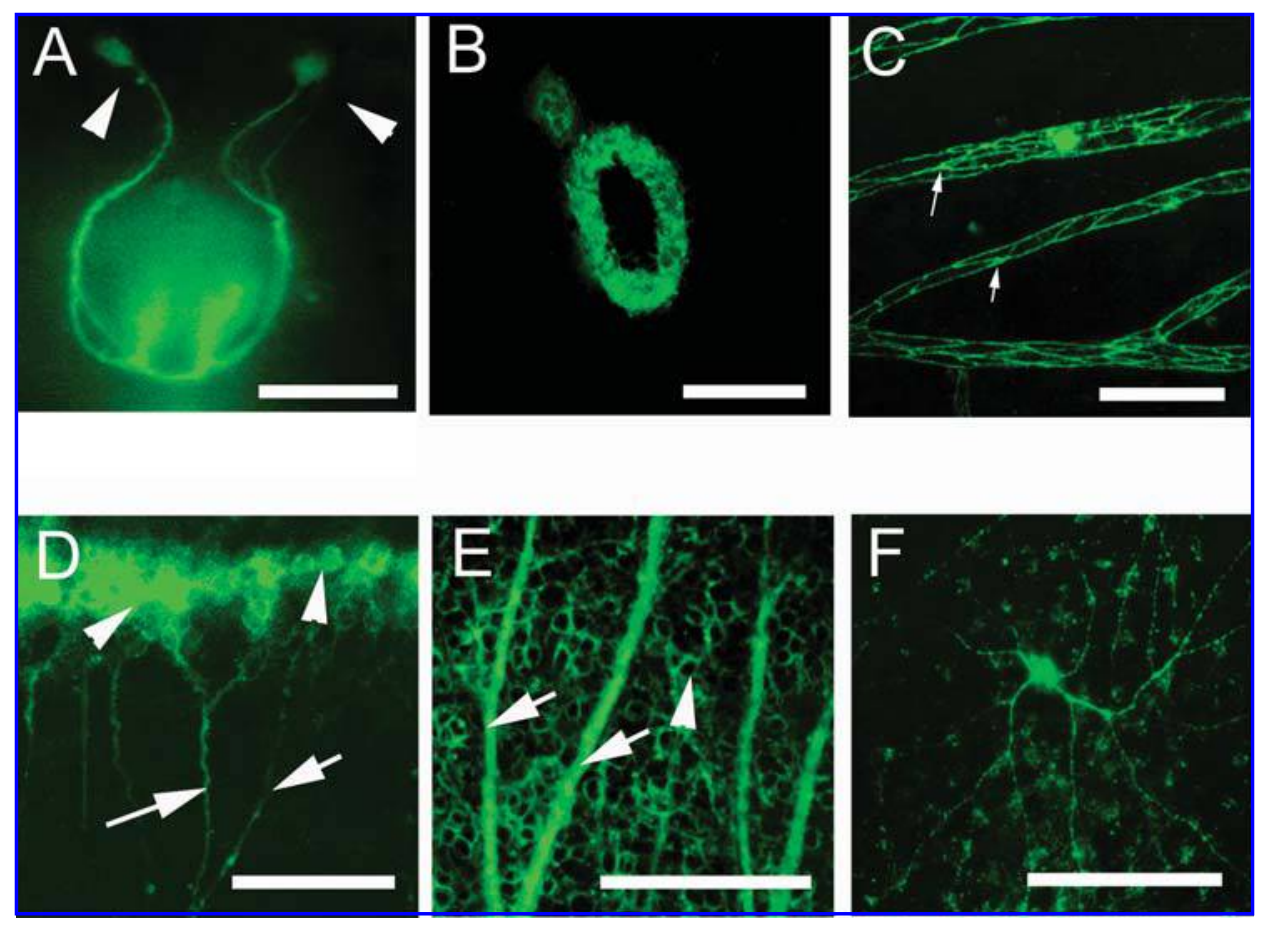

FIG. 5. Immunohistochemical localization of Thy-1 in adult goldfish nervous tissues. (A) Dorsal view on the rostral part of a goldfish brain labeled for Thy-1. From the olfactory bulbs (top), M802-positive cells (arrows) extend axons into the telencephalon. (D) and (E) Thy-1 immunoreactivity outlines endothelial cells of brain (B) and retinal (C) capillaries and is particularly intense at cell-cell contact sites (arrows). (D) In the normal retina, only the newborn retinal ganglion cells (arrowheads) at the margin of the retina and retinal axon fascicles (arrowheads) are labeled. (E) During axonal regeneration, however, Mab M802 labels all RGCs (arrows) and retinal axons heading towards the center of the retina (arrowhead). (F) In deeper retinal layers, some neurons which most likely represent amacrine cells, are stained. Where necessary, nuclei were counterstained with DAPI. Scale bars: $50 \mu \mathrm{m}$ for (B) to (F) and $500 \mu \mathrm{m}$ for (A).

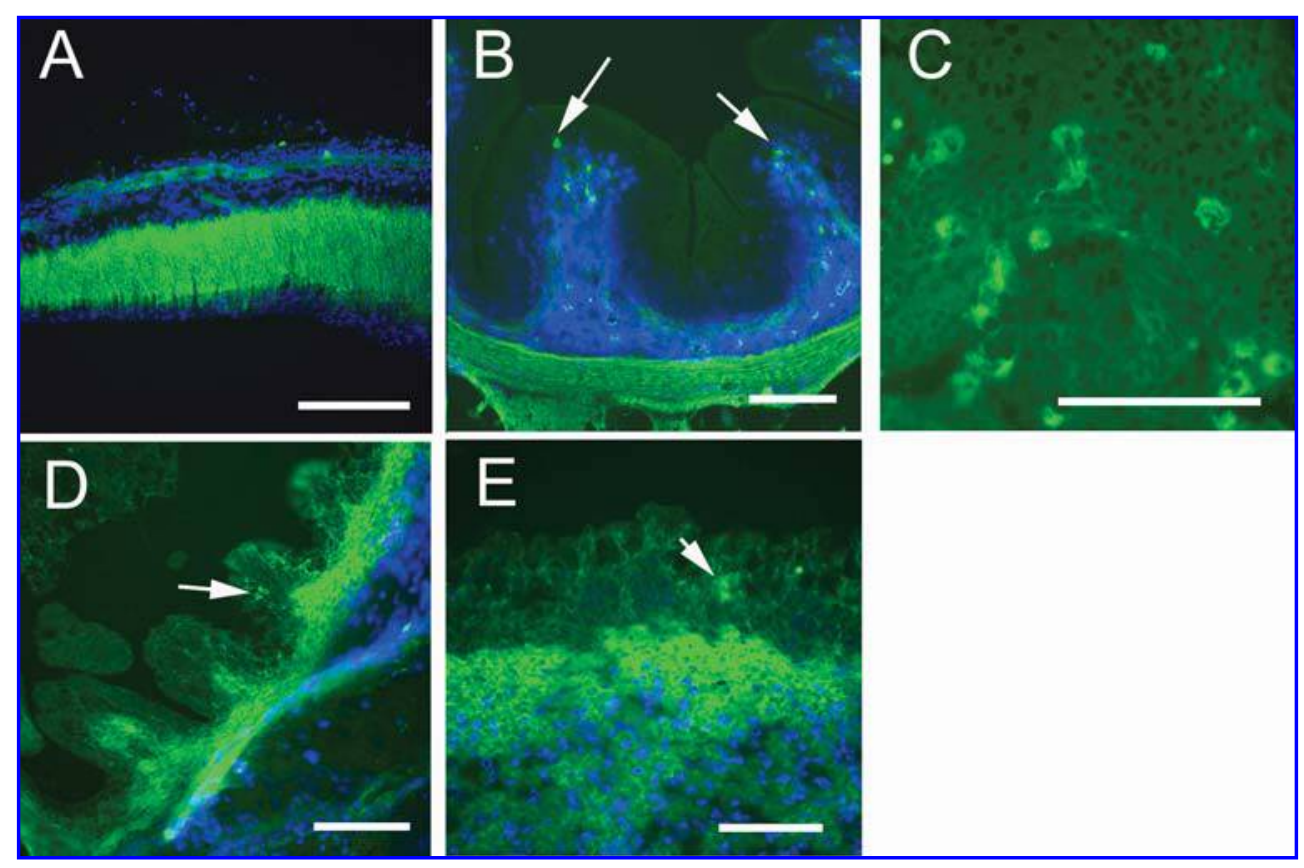

FIG. 6. Immunohistochemical localization of Thy-1 in adult nonneural tissues. Sections of several tissues labeled with M802 (green fluorescence) and counterstained with DAPI to visualize the nuclei where necessary. (A) The connective tissue in the swim bladder shows strong Thy-1 staining. (B) In the intestine, very strong label can be observed in the smooth muscle layer and in single cells of the mucosa (arrows). (C) In the kidney, single cells, most likely macrophages, are labeled by mAB M802. (D) In the gill arches, the cartilage and single cells (arrow) are labeled, as in the palatal organ (E). Scale bars : $50 \mu \mathrm{m}$ 
and the gill arches (Figs. 6D, 6E) and, to a lesser extent, the tubules of the kidney and the spleen (data not shown).

\section{DISCUSSION}

The aim of the present study was to characterize more closely the teleost Thy-1, homologues of which were known in mammals and birds for years without definitive proof of its function. We used antibody M802 to analyze the tissue distribution of the teleost Thy- 1 homolog, and bioinformatics to learn about its evolution and potential shift of function. Whereas the exact physiological role of Thy- 1 remains elusive, the high similarity among mammalian molecules has been suggestive of an important function with adaptive value for the organism. Not only polypeptide length and primary amino acid sequence are highly conserved (Fig. 1), but also gene coding structure and location in the genome. In contrast, fish and mammalian Thy1 are highly divergent in their amino acid sequences, and their homology relationship was originally inferred based on threshold levels of sequence and length similarity in database searches, as well as on the presence of a single Ig domain. ${ }^{5}$ In the present, more comprehensive study, we have extended our analysis with molecules from additional fish species and supported their homology to Thy-1 with additional criteria including gene structure and conservation of genomic location.

The rapid evolution of teleost Thy-1 sequences is evident in the accumulation of numerous amino acid substitutions relative to mammals; however, the general Ig fold as well as the number and disposition of $\alpha$-helical stretches are well conserved (Fig. 1). This evolutionary pattern was probably shaped by positive selection for substitutions which resulted in the development of new binding specificities, while still allowing for teleost Thy- 1 to retain the general properties and intrinsic roles of an Ig superfamily molecule. ${ }^{2}$ The functional consequences of these evolutionary changes need now to be experimentally verified.

At the genomic level, remarkable changes have also taken place around Thy-1 genes. We identified the zebrafish and Takifugu chromo- somal fragments homologous to the Thy- 1 region of mammals and found them to be duplicated. The syntenic relationships between the fish and mammalian genomic regions are not easily distinguishable because the fish chromosomal blocks have repeatedly undergone independent fission and gene loss events, breaking the conserved segment homology observed in mammals (Fig 3). Further analysis of the flanking intergenic regions should help to establish whether these chromosomal rearrangements also affected the expression and function of teleost Thy-1, for example, by placing these genes in other genomic environments under the direction of new regulatory elements, and driving their molecular evolution to the pace of novel selective pressures.

Nevertheless, at least part of the functions seem to be conserved between mammalian and teleost Thy-1, because Thy- 1 is reliably linked to the Reggie proteins in Jurkat T cells, PC12 cells as well as in goldfish fibroblasts. ${ }^{5,7}$ Additionally, Thy- 1 is associated with src and fyn kinases in neurons and $\mathrm{T}$ lymphocytes, suggesting that its function may only be uncovered when studied in the context of lipid rafts and its associated proteins that appear to form a "signalosome" for transmembrane communication.

In line with the species-specific differences in tissue distribution between mammals and birds, teleost Thy-1 shows its own distinct expression pattern. In both mouse and rat, Thy-1 is present at low levels at birth, but rises rapidly during the second and third weeks to reach adult levels at 3-4 weeks. ${ }^{27,28}$ This agrees with our observations in goldfish, where the Thy- 1 antigen could barely be detected at early embryonic stages. Expression in skin was detected at 3 days postfertilization, while expression in the nervous system occurred even later. The embryonic development in goldfish is not as tightly regulated as in zebrafish and varies with temperature and availability of food. Instead of defining stages by hours postfertilization, the size of the larvae turned out to be a good indicator of onset of Thy-1 expression. Expression of Thy-1 in the retina was detected in larvae of $12 \mathrm{~mm}$ length and larger, which corresponds approximately to an age of about 3 months under normal feeding conditions in our facilities. Interestingly, all RGCs express Thy-1 after op- 
tic nerve lesion, even those early RGCs born before the fish reached a length of $12 \mathrm{~mm}$, and which had not expressed Thy-1 at any previous stage of development.

In all vertebrate species studied in detail, including adult goldfish, the brain has been the only tissue in which Thy- 1 is consistently present in large amounts. ${ }^{29,30}$ In rodents, Thy-1 was detected in large amounts in the cerebral hemispheres, slightly less in brainstem, cerebellum, and spinal cord, and considerably less in peripheral nerves. ${ }^{28,30}$ In chicken, the highest Thy-1 concentration was seen in the forebrain which contains the highest density of synapses, followed by the midbrain, the brainstem, and spinal cord. ${ }^{23}$

The strongest expression in the goldfish was in brain regions dealing with sensory information processing (i.e., the vagal, facial, and glossopharyngeal lobes as well as in the vagal and facial nerves). The crucian carps, as most cyprinids, have an enormous number of taste buds in their mouth, the palatal organ, the gill region, and over the whole body surface. ${ }^{24}$ Taste buds, wherever they are located, are innervated by fibers from the VII, IX, and X cranial nerves. ${ }^{24}$ The vagal lobe is recipient of gustatory information sensed by the taste buds located in the intraoral cavity, whereas the facial lobe processes gustatory information perceived by taste buds on the lips and external skin of the head. ${ }^{31}$ In addition, changes in the intestinal microenvironment are sensed by vagal primary afferent fibers. ${ }^{32}$

Thus, the present staining pattern of Thy-1 in goldfish correlates well with the gustatory systems, and those organs where gustatory information is sensed are labeled: the palatal organ, the gill arches, the skin, cells in the intraoral cavity, and nerve cells in the intestine, as well as the nerves that transport the information (VII, IX, and X) to the medullary gustatory centers (vagal, facial, and glossopharyngeal lobes). In addition, subpopulation of neurons in the olfactory lobe that extend their axons into the telencephalon are also labeled intensely. Therefore, it seems that the sensory systems that are involved in finding and sorting of food are Thy- 1 positive in adults.

Outside the central nervous system, Thy-1 staining occurs in certain connective tissues such as the swim bladder and cartilage, in epithelia and blood vessels, the gill arches, and in specific cells in the palatal organ, kidney, and intestine. These findings are consistent with the tissue distribution of Thy-1 in higher vertebrates, where Thy- 1 has been detected in some, but not all collagen-containing connective tissues $^{33}$ such as kidney basement membrane, capillaries, and skin, as well as in nerve cells of the intestine and in different epithelia. However, the intensity and specific expression pattern varies considerably between species. Moderate staining of the teleost spleen is in accordance to the situation in rat, ${ }^{34}$ dogs, and humans, ${ }^{19}$ where also moderate amounts of Thy- 1 could be detected, but in contrast to the situation in sheep ${ }^{35}$ where Thy- 1 expression is reportedly strong. Strong staining of the basement membranes of the kidney tubules has been reported in rat and human, but not in mouse, ${ }^{33}$ whereas staining of the epidermis has been reported for human microvascular endothelium and perivascular dendritic cells ${ }^{36}$ as well as murine epidermis. ${ }^{37}$

Therefore, the findings outlined above are consistent with those reported for Thy-1 in other species and underline the developmental and tissue-specific regulation of Thy- 1 . They also predict a function of Thy- 1 in teleost sensory gustatory systems which may be an evolutionarily old function of Thy-1.

\section{ACKNOWLEDGMENTS}

The authors thank Dr. Christian Leppert for earlier contributions to this work.

\section{REFERENCES}

1. Cohen FE, Novotny J, Sternberg MJ, Campbell DG, Williams AF. Analysis of structural similarities between brain Thy-1 antigen and immunoglobulin domains. Evidence for an evolutionary relationship and a hypothesis for its functional significance. Biochem I 1981;195:31-40.

2. Williams AF, Gagnon J. Neuronal cell Thy-1 glycoprotein: homology with immunoglobulin. Science 1982; 216:696-703.

3. Campbell DG, Gagnon J, Reid KB, Williams AF. Rat brain Thy-1 glycoprotein. The amino acid sequence, 
disulphide bonds and an unusual hydrophobic region. Biochem I 1981;195:15-30.

4. Dowsing BJ, Gooley AA, Gunning P, Cunningham A, Jeffrey PL. Molecular cloning and primary structure of the avian Thy- 1 glycoprotein. Brain Res Mol Brain Res 1992;14:250-260.

5. Deininger SO, Rajendran L, Lottspeich F, Przybylski $\mathrm{M}$, Illges $\mathrm{H}$, Stuermer CAO, Reuter A. Identification of teleost Thy-1 and association with the microdomain/lipid raft reggie protins in regenerating CNS axons. Mol Cell Neurosci 2003;22:544-554.

6. Cooper, EL, Mansour MH. Distribution of Thy-1 in invertebrates and ectothermic vertebrates. Immunol Ser 1989;45:197-219.

7. Stuermer CAO, Lang DM, Kirsch F, Wiechers M, Deininger SO, Plattner H. Glycosylphosphatidyl inositol-anchored proteins and fyn kinase assemble in noncaveolar plasma membrane microdomains defined by reggie-1 and -2. Mol Biol Cell 2001;12:30313045.

8. Haeryfar SM, Al-Alwan MM, Mader JS, Rowden G, West KA, Hoski DW. Thy-1 signaling in the context of costimulation provided by dendritic cells provides signal 1 for $\mathrm{T}$ cell proliferation and cytotoxic effector molecule expression, but fails to trigger delivery of the lethal hit. LImmunol 2003;171:69-77.

9. Leyton L, Schneider P, Labra CV, Ruegg C, Hetz CA, Quest AF, Bron C. Thy-1 binds to integrin beta(3) on astrocytes and triggers foration of focal contact sites. Curr Biol 2001;11:1028-38.

10. Avalos AM, Labra CV, Quest AFG, Leyton L. Signalling triggered by Thy-1 interaction with beta(3) integrin on astrocytes is a essential step towards unraveling neuronal Thy-1 function. Biol Res 2002;35: 231-238.

11. Tiveron MC, Barboni E, Pliego Rivero FB, Gormley AM, Seeley PJ, Grosveld F, Morris R. Selective inhibition of neurite outgrowth on mature astrocytes by Thy-1 glycoprotein. Nature 1992;355:745-748.

12. Barlow JZ, Kelley KA, Bozdagi O, Huntley GW. Testing the role of the cell-surface molecule Thy-1 in regeneration and plasticity of connectivity in the CNS. Neuroscience 2002;111:837-852.

13. Morris RJ, Beech JN, Brber PC, Raisman G.. Early stages of Purkinje cell maturation demonstrated by Thy-1 immunohistochemistry on postnatal rat cerebellum. I Neurocytol 1985;14:427-452.

14. Morris RJ, Beech JN, Barber PC, Raisman G. Late emergence of Thy-1 on climbing fibers demonstrates a gradient of maturation from the fissures to the folial convexities in developing rat cerebellum. $[\mathrm{Neu}-$ rocytol 1985; 14:453-467.

15. Xue GP, Pliego Rvero B, Morris RJ. The surface glycoproten Thy- 1 is excluded from growing axons during development: a study of the expression of Thy-1 during axogenesis in hipocampus and brain. Development 1991;112:161-176.

16. Xue GP, Morris R. Expression of the neuronal surface glycoprotein Thy-1 does not follow appearance of its
mRNA in developing mouse Purkinje cells. I Neurochem 1992;58:430-440.

17. Raff MC. Surface antigenic markers for distinguishing $\mathrm{T}$ and $\mathrm{B}$ lymphocytes in mice. Transplant Rev 1971;6:52-80.

18. Acton RT, Morris RJ, Williams AF. Estimation of the amount and tissue distribution of rat Thy-1 antigen. Eur J Immunol 1974;4:598-602.

19. Dalchau R, Fabre JW. Identification and unusual tissue distribution of the canine and human homologues of Thy-1 (theta). LExp Med 1979;149:576-591.

20. Gordon JW, Chesa PG, Nishimura H, Rettig WJ, Maccari JE, Endo T, Seravalli E, Seki T, Silver J. Regulation of Thy-1 gene expression in transgenic mice. Cell 1987;50:445-452.

21. Schulte T, Paschke KA, Laessing U, Lottspeich F, Stuermer CA. Reggie-1 and reggie-2, two cell surface proteins expressed by retinal ganglion cells during axon regeneration. Development 1997;124:577-87.

22. Lang DM, Lommel S, Jung M, Ankerhold R, Petrausch B, Laessing U, Wiechers MF, Plattner H, Stuermer CA. Identification of reggie-1 and reggie-2 as plasma membrane-associated proteins which cocluster with activated GPI-anchored cell adhesion molecules in noncaveolar micropatches in neurons. L Neurobiol 1998;37:502-523.

23. Sinclair CM, Bartlett PF, Greig DI, Jeffrey PL. Distribution of Thy-1 in the avian nervous system: immunohistochemical and absorption analyses with a monoclonal antibody. Brain Res 1986;398:91-105.

24. Morita $Y$, Ito H, Masai H. Central gustatory paths in the crucian carp, Carassius carassius. L Comp Neurol 1980;191:119-132.

25. Morita Y, Murakami T, Ito H. Cytoarchitecture and topographic projections of the gustatory centers in a teleost, Carassius carassius. I Comp Neurol 1983;218: 378-394.

26. Morita Y, Finger T. Topographic and laminar organization of the vagal gustatory system in the goldfish, Carassius auratus. I Comp Neurol 1985;238:187-201.

27. Douglas TC. Occurrence of the theta-like antigen in rats. LExp Med 1972;136:1054-1062.

28. Reif AE, Allen JMV. The AKR thymic antigen and ist distribution in leukemias and nervous tissues. I Exp Med 1964;120:412-433.

29. Birnbaum G. Studies on brain-thymus cross-reactive antigens. Brain Res 1975;84:111-121.

30. Barclay AN, Hyden H. Localization of the Thy-1 antigen by immunofluorescence on neurons isolated from rat brain. L Neurochem 1979;32:1583-1586.

31. Atema J. Structures and functions of the sense of taste in the catfish. Brain Behav Evol 1971;4:273-294.

32. Ward SM, Bayguinov J, Won KJ, Grundy D, Berthoud, HR. Distribution of the vanilloid receptor (VR1) in the gastrointestinal tract. L Comp Neurol 2003;465:121-135.

33. Morris RJ, Ritter MA. Association of Thy-1 cell surface differentiation antigen with certain connective tissues in vivo. Cell Tissue Res 1980;206:459-475.

34. Acton RT, Pfeiffer SE. Distribution of Thy-1 differen- 
tiation alloantigen in the rat nervous system and a cell line derived from a rat peripheral neurinoma. Dev Neurosci 1978;1:110-117.

35. Sheldrake RF, Crocker BD, Husband AJ, Rostas JAP. Isolation and purification of a Thy-1-like glycoprotein from sheep brain and its distribution in ovine tissues. Res Vet Sci 1987;42:358-364.

36. Fivenson DP, Douglass MC, Nickoloff BJ. Cutaneous expression of Thy-1 in mycosis fungoides. Am J Pathol 1992;141:1373-1380.

37. Tschachler E, Schuler G, Hutterer J, Leibl H, Wolff K, Stingl G. Expression of Thy-1 antigen by murine epidermal cells. I Invest Dermatol 1983;81:282-285.

38. Geisler R, Rauch GJ, Baier H, van Bebber F, Bross L, Dekens MP, Finger K, Fricke C, Gates MA, Geiger H, Geiger-Rudolph S, Gilmour D, Glaser S, Gnugge L, Habeck H, Hingst K, Holley S, Keenan J, Kirn A,
Knaut H, Lashkari D, Maderspacher F, Martyn U, Neuhauss S, Neumann C, Nicolson T, Pelegri F, Ray R, Rick JM, Roehl H, Roeser T, Schauerte HE, Schier $A F$, Schoenberger U, Schoenthaler H-B, SchulteMerker S, Seydler C, Talbot WS, Weiler C, NuessleinVolhard C, Haffter P. A radiation hybrid map of the zebrafish genome. Nat Genet 1999;23:86-89.

Address reprint requests to: Alexander Reuter University of Konstanz Department of Biology 78457 Konstanz Germany

E-mail: Alexander.Reuter@uni-konstanz.de 



\section{This article has been cited by:}

1. L. Huang, T. Cheng, P. Xu, J. Duan, T. Fang, Q. Xia. 2009. Immunoglobulin superfamily is conserved but evolved rapidly and is active in the silkworm, Bombyx mori. Insect Molecular Biology 18:4, 517-530. [CrossRef]

2. 2005. Recent Papers on Zebrafish and Other Aquarium Fish ModelsRecent Papers on Zebrafish and Other Aquarium Fish Models. Zebrafish 1:4, 369-375. [Citation] [PDF] [PDF Plus] 